\title{
Attention, discrimination learning, and observing responses'
}

DALBIR BINDRA AND ALAN MOSCOVITCH

MCGILL UNIVERSITY

\begin{abstract}
The extent of novelty reactions and of disruption of a running response, brought about by stimulus changes made at cue-positions located at different distances from the reward, were measured in 12 rats. Both measures showed higher values for the cue-position closer to the reward. The results support the idea of a general attentiveness factor that is distinct from observing responses which might emerge as a consequence of differential responding.
\end{abstract}

\section{Problem}

The concept of "observing responses" was introduced by Wyckoff (1952) as a variable responsible for some perceptual selectivity in discrimination tasks. The development of observing responses is considered by Wyckoff to be a function of the extent to which $\mathrm{S}$ responds differently to discriminative stimuli; differential responding is assumed to be necessary for reinforcing observing responses. Thus he regards the emergence of any specific set of observing responses to be linked to particular discriminative stimuli. However, it seems reasonable to suppose that some additional, nonspecific attentional factor might also play a part in the early stages of discrimination learning. In the present study we investigated the possible existence of such a general attentional factor that might precede the emergence of differentiated observing responses.

Raskin (1965) has shown that successive discrimination learning in a runway is inversely related to the degree of cue-reward separation. On the assumption of a general attentional factor, one would expect Raskin's Ss to have attended differentially to stimuli at the two cue-position areas regardless of the success or failure of discrimination learning and of the presence or absence of discriminative stimuli. In the present experiment we examined this possibility by studying "novelty reactions" (rearing, sniffing, etc.) made by rats in response to stimulus changes introduced at the two cue-positions in the same discrimination apparatus as was used by Raskin. The extent of increase in novelty reactions following a stimulus change at a cue-position would indicate the degree to which the animals had attended to the original stimuli at that position. We expected a stimulus change in the near cue-position to evoke novelty reactions and interfere with the running response even when Ss had been given discrimination training (unsuccessfully) with cues in the position far from the goal.

\section{Method}

The Ss were 12 naive male hooded rats, about 100 days old. They were maintained on a 23-hr water deprivation schedule.

The apparatus was the same as that described by Raskin (1965). Essentially, it was an 18-in runway connected to a 12-in start box and a 14-in goal box containing a water tube. Stimulus cards were presented at two cue-positions, at the entrance of the runway ("far") and at the entrance to the goalbox ("near"); Ss had to push the cards and go under them. No separate starting gate was employed. Starting and running times were measured.

Following two days of preliminary training, 10 discrimination trials, five rewarded and five nonrewarded, were given each day in a scrambled order. Discrimination cues were presented only at the far cue-position; a black stimulus card was presented on rewarded trials and a white stimulus card on nonrewarded trials. A grey card was present at the near cue-position on every trial; it did not serve as a differential cue.

Stage 1 of the experiment consisted of 340 training trials. On the final 50 of these trials, S's responses in the start box and running area were observed (see Bindra \& Spinner, 1958; Claus \& Bindra, 1960). If a response occurred which was not related to approaching the goal box directly (e.g., rearing, sniffing, scratching, biting, etc.) a "novelty reaction score" of 1 was recorded for that trial. If no such reaction was observed, a score of 0 was given.

In Stage 2, the positions of the stimulus cards were reversed. That is, the grey stimulus card was now presented at the far cue-position, and the black or white stimulus card, still denoting the same reinforcement contingency, was presented at the near cue-position. Sixty further discrimination trials were given with starting time, running time, and novelty reactions being recorded as before.

\section{Results}

Difference scores (median time on nonrewarded trials minus median time on rewarded trials) were calculated for starting and running times for each day separately. Median difference scores for blocks of three days (30 trials) were averaged over the 12 Ss. Figure 1 presents these results。

As expected (Raskin, 1965), none of the animals learned the discrimination problem in Stage 1, with the discrimination cues in the far position.

As soon as the positions of the cue cards were interchanged (Day 1 of Stage 2), running times increased. Mean running times for the first two trials of the last day of Stage 1 and for the first two trials of the 


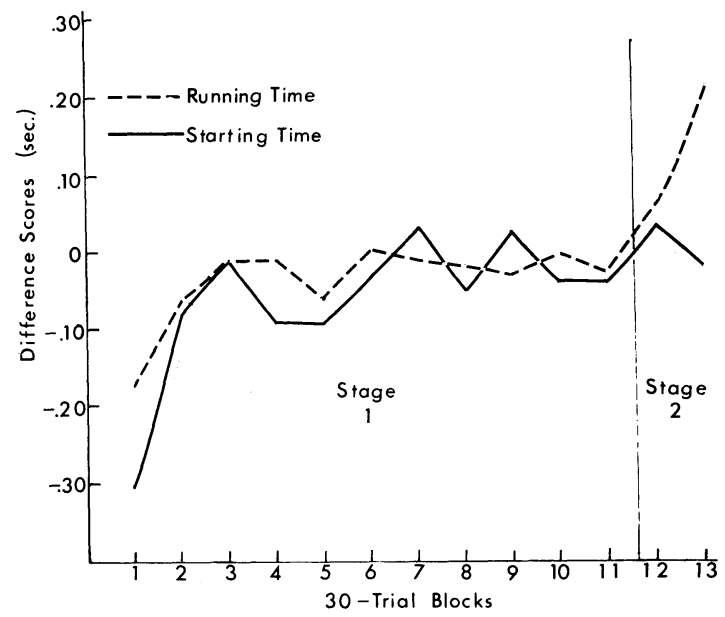

Fig. 1. Average median running and starting time difference scores for 12 Ss. Stage 1: cue at "far" position; Stage 2: cue at "near" position. (Block 11 consisted of 40 trials.)

first day of Stage 2 were determined for each S. (On both days, the first trial was rewarded, the second, nonrewarded.) The difference between these two sets of scores was found, by a sign test, to be significant at the .05 level. Correspondingly, while not a single novelty reaction was observed in the running area during the last day of Stage 1 , nine such reactions were recorded on the first day of Stage 2 .

The interchange of stimulus cards at the far cueposition was not accompanied by any changes in start box behavior. There was no marked difference in novelty reactions (58 on the last day of Stage 1, 52 on the first day of Stage 2), nor was there any noticeable difference in the mean starting times for the first two trials of the last day of Stage 1 and of the first day of Stage 2 .

In Stage 2, with discrimination cues now at the near position, a tendency toward longer running times in the presence of the negative cue became evident. The median running time difference scores on the lastblock of trials in Stage 2 were significantly longer than those on the last block of trials in Stage 1 (Wilcoxon matched-pairs signed ranks test, $p<.05$ ), indicating the beginning of discrimination learning.

\section{Diseussion}

Since, in Stage 1 , the rats failed to respond differentially to the discriminative cues, presumably no observing responses of the type described by Wyckoff (1952) could have developed. That some other attentional factor was nevertheless operative is shown by the fact that a stimulus change in the near cue-position reliably affected behavior. Clearly, during training, Ss must have attended to the grey card in the near cue-position; only then could the stimulus change in that position have produced novelty reactions and response disruption. This, coupled with Raskin's (1965) findings that suc- cessful discrimination learning occurred only when the cues were in the near position, indicates that Ss attended more to stimuli at the near position than to stimuli at the far position.

These findings imply the existence of a gradient of attentiveness such that attention to stimuli decreases with increasing distance from the reward (or with other factors related to spatial separation). Such a conclusion must, however, remain tentative until it is shown that a reversal of cues would produce the same results even if, during training, the discriminative cues were to be presented in the near position. This condition could not be examined in the present experiment for, if cue cards had been presented in the near cue-position, successful discrimination learning would have been a confounding factor. Decisive evidence on the possible existence of an attentiveness gradient could perhaps be obtained in a non-discriminative learning situation.

One possible interpretation of the proposed gradient is that the attending response, like other responses, habituates in the absence of reward. Therefore, owing to the operation of a delay of reward gradient, the attending response would be maintained closer to the reward while quickly habituating in other areas of the apparatus. It follows that stimuli near the reward would be more readily noted. If we further assume that the neural traces representing stimuli that have been attended to remain salient longer than those of stimuli that have not been attended to, then the chances of a cue trace being active when the animal encounters the outcome (reward or nonreward) will be greater in the case of cues closer to the reward. Hence the probability of successful discrimination learning, and of the emergence of observing responses, would also be greater. The gradients of visual sampling (Schuck, 1960) and observing responses (Stollnitz, 1965), proposed to explain the effects of cue-response separation on discrimination learning, may result from the operation of an attentiveness gradient.

\section{References}

Bindra, D., \& Spinner, N. Response to different degrees of novelty: The incidence of various activities. J. exp. Anal. Behav., 1958, 1, 341-350.

Claus, H. J., \& Bindra, D. Reactions to novelty and stimuluschange induced response decrement. Canad. J. Psychol., 1960, $14,101-110$.

Raskin, L. M. The influence of cue position and pretraining on successive discrimination learning in the rat. Psychon. Sci., 1965, 3, 221-222.

Schuck, J. R. Pattern discrimination and visual sampling by the monkey. J. comp. physiol. Psychol., 1960, 53, 251-255.

Stollnitz, F. Spatial variables, observing responses, and discrimination learning sets. Psychol. Rev., 1965, 72, in press

Wyckoff, L. B., Jr. The role of observing responses in discrimination learning: Part I. Psychol. Rev., 1952, 59, 431-442.

Note

1. This research was supported by Grant No. APT-74 from the National Research Council of Canada and Grant No. MH-03238 from the United States Public Health Service. 